\title{
Advances in the Treatment of Endometrial and Ovarian Cancer
}

\author{
An Expert Interview with David G Mutch
}

Washington University School of Medicine, St Louis, MO, USA

DOI: https://doi.org/10.17925/OHR.2019.15.1.23

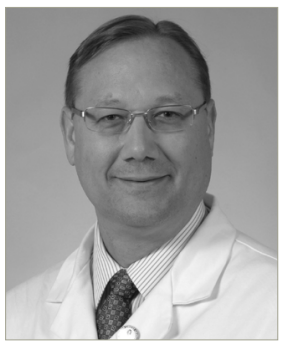

\section{David G Mutch}

Dr Mutch is the Judith and Ira Gall Professor of Obstetrics and Gynecology and the Director of Gynecologic Oncology at the Washington University School of Medicine in St Louis, MO, USA. He is a member of the Division of Gynecologic Oncology of the American Board of Obstetrics and Gynecology, and serves as a reviewer for multiple medical journals related to women's health and cancers of the female reproductive tract. He is a member of the American Osteopathic Association and is a past president of the Society of Gynecologic Oncology. Dr Mutch serves as the Chair of the Barnes Jewish Hospital Cancer committee and is the leader of the Gynecological Focus group for the Siteman Cancer Center.

\section{Keywords}

Endometrial cancer, ovarian cancer, PARP inhibitors, DNA mismatch repair, BRCA1, BRCA2

Disclosures: David G Mutch has nothing to disclose in relation to this article.

Acknowledgments: Medical writing assistance was provided by Katrina Mountfort of Touch Medica Media, and funded by Touch Medical Media.

Review process: This is an expert interview and, as such, has not undergone the journal's standard peer review process.

Authorship: The named author meets the International Committee of Medical Journal Editors (ICMJE) criteria for authorship of this manuscript, takes responsibility for the integrity of the work as a whole, and has given final approval to the version to be published.

Received: December 3, 2018

Published Online: April 4, 2019

Citation: Oncology \& Hematology Review. 2019;15(1):23-4

Corresponding Author: David G Mutch, Washington University School of Medicine, 660 South Euclid Avenue, St Louis, MO 63110, USA. E: mutchd@wustl.edu.

Support: No funding was received in the publication of this article.
G ynecological malignancies can be difficult to treat. Ovarian cancer carries with it the worst prognosis of all gynecological cancers. Approximately $75 \%$ of patients with epithelial ovarian cancer are diagnosed with advanced disease which is curable only in a minority of cases, resulting in a 5-year overall survival rate of only $20-30 \%$. However, survival at all stages is improving. Conversely, incidence and mortality is increasing in patients with endometrial cancer., ${ }^{2,3}$

The recent approval of poly (ADP-ribose) polymerase (PARP) inhibitors for ovarian cancer and the enthusiasm regarding the use of immune checkpoint inhibitors for all gynecological malignancies continue to broaden the treatment landscape. In this expert interview, David Mutch discusses the latest advances in the treatment of endometrial and ovarian cancer.

\section{Q. What are the greatest unmet needs in the management of endometrial and ovarian cancer?}

The unmet needs in endometrial cancer are that we have not been able to identify treatments that make a significant difference in survival. Furthermore, the incidence and mortality are increasing 2,3 and we don't know why. In terms of gynecological research, endometrial cancer is a high priority. In terms of ovarian cancer, patients are living with their disease longer. ${ }^{1}$ We are successful in the treatment and management of these patients, but we are still not preventing the disease. Though we are helping patients live longer with their disease, we are not necessarily curing more people. We need methods for predicting mortality through genetic evaluation and screening, but this is some distance away.

\section{Q. What have been the major recent advances in targeted therapy for endometrial cancer?}

We now know that around 30\% of patients have a DNA mismatch repair deficiency, either due to an inherited germline mutation or a somatic mutation where the mismatch repair gene is silenced by methylation. ${ }^{5}$ These patients can be targeted with programmed cell death 1 (PD-1) inhibitors, most notably pembrolizumab ${ }^{6,7}$ or nivolumab. ${ }^{8}$ 


\section{Q. Could you tell us a little about PARP inhibitors and their impact on the management of ovarian cancer?}

There are currently three PARP inhibitors - olaparib, rucaparib and niraparib - approved by the US Food and Drug Administration for commercial use in the treatment of ovarian cancer.9-11 These have become a useful addition to our treatment armamentarium for ovarian cancer and have been extremely successful. Those patients who benefit most are patients who have homologous recombination deficiency or whose tumor has a homologous recombination deficiency, the most common being BRCA1 and BRCA2 mutations. However, we are learning that other patients without apparent mutations may also benefit. Furthermore, patients are now benefiting in the maintenance setting of upfront therapy. Other patients benefit for reasons we don't understand, possibly PARP trapping or other mechanisms. Many patients will benefit from PARP inhibitor therapy and this is translating to improved survival.

\section{Q. Which patients are most likely to benefit from PARP inhibitors?}

Patients with homologous recombination deficiency are the ones that will benefit the most. This is most commonly due to BRCA1/2 mutations. However, there are patients who have mutations in other DNA repair genes, such as RAD50, who will also benefit. ${ }^{12}$ As we learn more, we will be able to better identify patients most likely to respond.

\section{Q. What do you think are the next steps in the development of these therapies?}

Ongoing trials are investigating a number of questions about the clinical use of PARP inhibitors in ovarian cancer, including their use as first-line treatment as well as other targeted therapies. The next steps are to better understand the driver mutations influencing what allows patients to respond to chemotherapy and/or targeted therapy. At the moment, 'one size fits all' is the standard approach, but we would rather tailor therapy to the individual. The PARP inhibitors and immune checkpoint inhibitors represent the tip of the iceberg; I would expect many new therapies to emerge in the coming years.

\section{Q. What do you think are the next steps in the treatment of endometrial cancer?}

We are trying to understand the importance of lymph node involvement and the best treatment for those patients. We are also trying to understand the importance of immunotherapy in this group of patients. Sadly, endometrial cancer is increasing in incidence and mortality, and we need to understand this better and the reasons for it. In order to do that, we need to understand the molecular genesis of this disease so that we can better target the specific abnormalities that occur within this disease. $\square$
1. Wright JD, Chen $L$, Tergas Al, et al. Trends in relative survival for ovarian cancer from 1975 to 2011. Obstet Gynecol. 2015;125:1345-52.

2. Bray F, Ferlay J, Soerjomataram I, et al. Global cancer statistics 2018: GLOBOCAN estimates of incidence and mortality worldwide for 36 cancers in 185 countries. CA Cancer J Clin. 2018;68:394-424.

3. Lortet-Tieulent J, Ferlay J, Bray F, et al. International patterns and trends in endometrial cancer incidence, 1978-2013. J Natl Cancer Inst. 2018;110:354-61

4. Kato $\mathrm{M}$, Takano $\mathrm{M}$, Miyamoto $\mathrm{M}$, et al. DNA mismatch repairrelated protein loss as a prognostic factor in endometrial cancers. J Gynecol Oncol. 2015;26:40-5.

5. Masuda K, Banno K, Yanokura M, et al. Relationship between DNA mismatch repair deficiency and endometrial cancer. Mol Biol Int 2011;2011:256063.

6. Le DT, Uram JN, Wang H, et al. PD-1 Blockade in tumors with mismatch-repair deficiency. N Engl J Med. 2015;372:2509-20.

7. Ott P, Bang Y-J, Berton-Rigaud D, et al. Safety and antitumor activity of pembrolizumab in advanced programmed death ligand 1-positive endometrial cancer: results from the KEYNOTE-028 study. J Clin Oncol. 2017;35:2535-41.

8. Castellano T, Moore KN, Holman LL. An overview of immune checkpoint inhibitors in gynecologic cancers. Clin Ther. 2018;40:372-88.

9. Pujade-Lauraine E, Ledermann JA, Selle F, et al. Olaparib tablets as maintenance therapy in patients with platinum-sensitive, relapsed ovarian cancer and a BRCA1/2 mutation (SOLO2/ENGOT-OV21): a double-blind, randomised, placebo-controlled, phase 3 trial. Lancet Oncol. 2017;18:1274-84.

10. Mirza MR, Monk BJ, Herrstedt J, et al. Niraparib maintenance therapy in platinum-sensitive, recurrent ovarian cancer. N Eng/ J Med. 2016;375:2154-64.

11. Swisher EM, Lin KK, Oza AM, et al. Rucaparib in relapsed, platinum-sensitive high-grade ovarian carcinoma (ARIEL2 Part 1): an international, multicentre, open-label, phase 2 trial. Lancet Oncol. 2017; 18:75-87.

12. Konstantinopoulos PA, Ceccaldi R, Shapiro GI, et al. Homologous recombination deficiency: exploiting the fundamental vulnerability of ovarian cancer. Cancer Discov. 2015;5:1137-54. 